\title{
Efeitos hemodinâmicos da anestesia em plano profundo com infusão intravenosa contínua de propofol ou propofol associado à lidocaína em cães
}

\author{
Hemodynamic effects of deep anesthesia with a constant rate infusion \\ of propopol or propofol combined with lidocaine in dogs
}

\author{
Rodrigo Mannarino ${ }^{\mathrm{I}}$ Stelio Pacca Loureiro Luna ${ }^{\mathrm{I}}$ Eduardo Raposo Monteiro ${ }^{\mathrm{I}, \mathrm{II}}{ }^{*}$ \\ Sara Maria de Carvalho e Suzano ${ }^{\text {III }}$ Thais Feres Bressan ${ }^{\text {II }}$
}

RESUMO

\begin{abstract}
Os efeitos hemodinâmicos da anestesia total intravenosa com propofol ou propofol associado à lidocaína foram estudados em 12 cães. No grupo $P(n=6)$, os animais receberam bolus de $6 \mathrm{mg} \mathrm{kg}^{-1}$ de propofol e infusão contínua de $1,25 \mathrm{mg} \mathrm{kg}^{-1}$ $\mathrm{min}^{-1}$. No grupo PL $(n=6)$, os animais receberam bolus de $6 \mathrm{mg}$ $\mathrm{kg}^{-1}$ de propofol e $1,5 \mathrm{mg} \mathrm{kg}^{-1}$ de lidocaína, seguido de infusão de 1,0mg $\mathrm{kg}^{-1} \mathrm{~min}^{-1}$ e $0,25 \mathrm{mg} \mathrm{kg}^{-1} \mathrm{~min}^{-1}$, dos mesmos fármacos, respectivamente. Os animais foram instrumentados para mensuração das variáveis hemodinâmicas e do indice bispectral (BIS), aos 75, 90, 105 e 120 minutos de anestesia. Foram observados valores menores de indice cardíaco, indice sistólico, pressões arteriais sistólica, diastólica e média no grupo $P$ do que no grupo $P L(P<0,05)$. Não foram observadas diferenças entre os grupos na frequência cardíaca, índice de resistência vascular sistêmica e BIS. As concentrações plasmáticas de propofol foram menores no grupo PL do que no grupo $P$ (medianas de 5,7 a

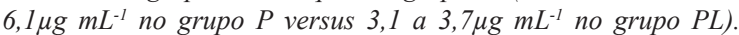
As concentrações plasmáticas de lidocaína (medianas de 2,27 a 2,51 $\mu \mathrm{g} \mathrm{mL}^{-1}$ ) mensuradas encontram-se na faixa que resulta em analgesia e abaixo de valores que resultam em toxicidade em cães. Os valores de BIS obtidos nos dois grupos foram compativeis com plano profundo de anestesia (médias de 43 a 46 e 45 a 49 nos grupos P e PL, respectivamente). A manutenção da anestesia em plano profundo com lidocaina-propofol causa menor depressão cardiovascular do que a anestesia com dose equipotente de propofol isoladamente.
\end{abstract}

Palavras-chave: anestesia intravenosa, eletroencefalografia, sistema cardiovascular, anestesia balanceada.

\section{ABSTRACT}

The hemodynamic effects of total intravenous anesthesia with propofol or propofol in combination with lidocaine were investigated in 12 dogs. In the P group $(n=6)$, the dogs received a loading dose (LD) of $6 \mathrm{mg} \mathrm{kg}^{-1}$ of propofol followed by a constant rate infusion (CRI) of $1.25 \mathrm{mg} \mathrm{kg}^{-1} \mathrm{~min}^{-1}$. In the PL group $(n=6), \operatorname{dogs}$ received a $L D$ of $6 \mathrm{mg} \mathrm{kg}^{-1}$ of propofol and $1.5 \mathrm{mg} \mathrm{kg}^{-1}$ of lidocaine followed by CRIs of $1.0 \mathrm{mg} \mathrm{kg}^{-1} \mathrm{~min}^{-1}$ and $0.25 \mathrm{mg} \mathrm{kg}^{-1} \mathrm{~min}^{-1}$ of propofol and lidocaine, respectively. The animals were instrumented for measurement of hemodynamic variables and bispectral index (BIS), recorded at 75, 90, 105 and 120 minutes during anesthesia. Cardiac index, stroke index, systolic, diastolic and mean arterial blood pressures were lower in the P group compared to the PL group $(P<0.05)$. There were no significant differences between groups in heart rate, systemic vascular resistance index and BIS. Plasma concentrations of propofol were lower in group PL than in group $P$ (medians of 5.7 to $6.1 \mathrm{mg} \mathrm{mL}^{-1}$ in the $P$ group versus 3.1 to $3.7 \mathrm{mg}$ $m L^{-1}$ in the PL group). Measured lidocaine plasma concentrations (medians of 2.27 to $2.51 \mathrm{mg} \mathrm{mL}^{-1}$ ) were in the range that result in analgesia and were below values that result in toxicity in dogs. The BIS values observed in the two groups of dogs were compatible with deep anesthesia (mean values of 43-46 and 45-49 in groups $P$ and $P L$, respectively). Maintenance of deep anesthesia with lidocainepropofol causes less cardiovascular depression than equipotent doses of propofol alone.

Key words: anesthesia, intravenous, electroencephalography, cardiovascular system, balanced anesthesia.

\section{INTRODUÇÃO}

O uso da anestesia total intravenosa (ATIV) tem sido incrementado como alternativa ao uso de anestésicos inalatórios e, nesse contexto, o propofol é um dos fármacos mais utilizados para a manutenção desta modalidade de anestesia

\footnotetext{
'Departamento de Cirurgia e Anestesiologia Veterinária, Faculdade de Medicina Veterinária e Zootecnia (FMVZ), Universidade Estadual Paulista (UNESP), Botucatu, SP, Brasil.

IIPrograma de Pós-graduação em Ciência Animal, Universidade Vila Velha (UVV), Rua Comissário José Dantas de Melo, 21, 29102-770, Boa Vista, Vila Velha, ES, Brasil. E-mail: btraposo@hotmail.com.*Autor para correspondência.

IIICurso de Medicina Veterinária, Universidade Castelo Branco (UCB), Rio de Janeiro, RJ, Brasil.
} Recebido 04.09.12 Aprovado 26.09.13 Devolvido pelo autor 06.12.13 CR-2012-0798.R2 
(KEEGAN \& GREENE, 1993; HALL et al., 1997; MANNARINO et al., 2012). Os efeitos do propofol sobre a nocicepção são discutíveis e não completamente esclarecidos, sendo relatada ausência de ação antinociceptiva (FRÖLICH et al., 2005) bem como efeito antinociceptivo e anti-hiperalgésico (TAKECHI et al., 2013). Por essa razão, o propofol não é recomendado como agente único e deve ser associado a agentes analgésicos para a manutenção da ATIV em procedimentos cirúrgicos. Outra desvantagem é que esse agente é cardiodepressor e, em cães, causa redução na contratilidade do miocárdio (PAGEL \& WARLTIER, 1993), na pressão arterial (PAGEL \& WARLTIER, 1993; MANNARINO et al., 2012) e no débito cardíaco (MANNARINO et al., 2012). A associação de fármacos antinociceptivos com o propofol, além de proporcionar analgesia intraoperatória, possibilita reduzir a dose de manutenção e a depressão cardiovascular do propofol.

A infusão contínua de lidocaína tem sido utilizada em associação aos anestésicos inalatórios, na manutenção da anestesia. Esse fármaco causa redução dose dependente da concentração alveolar mínima (CAM) dos anestésicos inalatórios em cães, sem causar efeitos adversos sobre a frequência cardíaca e a pressão arterial (VALVERDE et al., 2004). Seu uso em associação ao propofol na manutenção da anestesia também foi relatado em cães. A administração de lidocaína na dose de 0,25 $\mathrm{mg} \mathrm{kg}^{-1} \mathrm{~min}^{-1}$ reduziu de forma não significativa em 18\% a taxa de infusão mínima (TIM) do propofol. Entretanto, durante a manutenção da anestesia em plano superficial, empregando 1 TIM, não se observaram benefícios hemodinâmicos da anestesia com propofol associado à lidocaína, em comparação ao uso isolado do propofol (MANNARINO et al., 2012). Considerando-se que a anestesia em plano superficial é inadequada para procedimentos cirúrgicos, não é possível afirmar se os resultados de MANNARINO et al. (2012) seriam reproduzidos em plano profundo de anestesia, situação em que se espera maior depressão cardiovascular pelo propofol. Dessa forma, o presente estudo objetivou comparar os efeitos hemodinâmicos da anestesia em plano profundo, com propofol isolado ou associado à lidocaína.

\section{MATERIAL E MÉTODOS}

Foram utilizados 12 cães hígidos machos, com idade entre 2 e 5 anos, sem raça definida, com peso de $12,3 \pm 1,4 \mathrm{~kg}$ (média $\pm \mathrm{DP})$. Os animais foram considerados hígidos, baseado na ausência de anormalidades em exames físico e laboratoriais (hemograma, ureia, creatinina, fosfatase alcalina e aspartato amino transferase). Após jejum alimentar prévio de 12 horas e hídrico de duas horas, foi realizada cateterização venosa através da introdução de um cateter 20 ou $22 \mathrm{G}$ na veia cefálica direita.

Os animais foram aleatoriamente divididos em dois grupos de seis animais cada e tratados por via IV com $6 \mathrm{mg} \mathrm{kg}^{-1}$ de propofol $^{\text {a }}$ para indução e infusão contínua de $1,25 \mathrm{mg} \mathrm{kg}^{-1} \mathrm{~min}^{-1}$ para manutenção da anestesia (Grupo P) ou $6 \mathrm{mg} \mathrm{kg}^{-1}$ de propofol, associado a $1,5 \mathrm{mg} \mathrm{kg}^{-1}$ de lidocaína ${ }^{\mathrm{b}}$ para indução e manutenção da anestesia com infusão contínua de propofol associado à lidocaína, nas doses de 1,0mg $\mathrm{kg}^{-1} \mathrm{~min}^{-1}$ e $0,25 \mathrm{mg} \mathrm{kg}^{-1} \mathrm{~min}^{-1}$, respectivamente (Grupo $\mathrm{PL})$, por meio de bomba de infusão de seringa ${ }^{\mathrm{c}}$.

Após a indução da anestesia, os animais foram posicionados em decúbito dorsal sobre um colchão térmico e foi realizada a intubação orotraqueal com uma sonda com cuff, a qual foi conectada a um circuito circular valvular para fornecimento de oxigênio $100 \%\left(2 \mathrm{~L} \mathrm{~min}{ }^{-1}\right)$. Foi instituída a ventilação artificial $^{\mathrm{d}}$ com pressão de pico das vias aéreas de $10 \mathrm{a}$ $15 \mathrm{~cm} \mathrm{H}_{2} \mathrm{O}$ e a frequência respiratória $\left(f_{\mathrm{R}}\right)$ foi ajustada para manter a concentração expirada de dióxido de carbono $\left(\mathrm{ETCO}_{2}\right)$ entre 30 e $40 \mathrm{mmHg}^{\mathrm{e}}$. Um cateter $20 \mathrm{G}$ foi introduzido por punção percutânea na artéria femoral e conectado a um transdutor de pressão ${ }^{f}$ previamente zerado na altura do manúbrio do esterno, para mensuração da pressão arterial sistólica (PAS), diastólica (PAD) e média (PAM). A frequência cardíaca (FC) e ritmo cardíaco foram monitorados utilizando a derivação DII de um eletrocardiograma ${ }^{\mathrm{e}}$. A temperatura foi monitorada utilizando um termômetro esofágico ${ }^{\mathrm{e}}$.

Um cateter introdutor $6 \mathrm{~F}^{\mathrm{g}}$ foi introduzido na veia jugular direita e suturado à pele, para permitir a introdução do cateter Swan-Ganz $5 \mathrm{~F}^{\mathrm{h}}$, cuja extremidade distal foi posicionada na artéria pulmonar. O correto posicionamento do cateter de Swan-Ganz foi verificado pela observação das ondas de pressão características no monitor multiparamétrico ${ }^{\mathrm{e}}$. O cateter de Swan-Ganz foi utilizado para mensuração do débito cardíaco (DC), pressão média da artéria pulmonar (PAP), pressão de oclusão da artéria pulmonar (POAP) e pressão venosa central (PVC). O DC foi mensurado por meio de termodiluição, cujo valor foi obtido em cada momento pela média de três mensurações, com diferença inferior a $10 \%$ entre si. A partir dos valores hemodinâmicos, foram calculados os índices cardíaco (IC), sistólico (IS) e de resistência vascular sistêmica e pulmonar (IRVS e IRVP) por meio de equações relatadas na literatura (HASKINS et al., 2005). 
Para monitoração do BIS, foi empregado o monitor A-2000 XPi (versão de software 3.3). Após a tricotomia da região encefálica dos animais e limpeza com éter, o sensor para a captação dos sinais eletroencefalográficos foi fixado na configuração frontal-temporal (CAMPAGNOL et al., 2007). Os valores de BIS com índice de qualidade do sinal (SQI) abaixo de 50, numa escala de 0 a 100 , foram rejeitados.

Após o término do período de instrumentação de 60 minutos, as variáveis anteriormente descritas foram mensuradas em intervalos de 15 minutos, por 60 minutos (momentos $75,90,105$ e 120 minutos). Simultaneamente, amostras de sangue arterial foram colhidas em tubos com EDTA e refrigeradas a $-4^{\circ} \mathrm{C}$. Após a última coleta (120 minutos), as amostras foram centrifugadas, o plasma foi extraído e armazenado a $-20^{\circ} \mathrm{C}$ para mensuração das concentrações plasmáticas de propofol e lidocaína por meio da Cromatografia Líquida de Alta Performance, conforme descrito anteriormente por VIEIRA (1999) e DOHERTY \& FRAZIER (1998), respectivamente. Os limites de detecção e coeficientes de variação intra-ensaio e inter-ensaio foram: propofol, $0,1 \mu \mathrm{g} \mathrm{mL}^{-1}, 6,2 \%$ e $7,5 \%$; lidocaína, $0,043 \mu \mathrm{g} \mathrm{mL}^{-1}, 3,7 \%$ e 5,4\%. Após o registro das variáveis aos 120 minutos, a administração de anestésicos foi interrompida. A recuperação da anestesia foi acompanhada e registrados os tempos decorridos até a extubação, decúbito esternal e posição quadrupedal dos animais.

A distribuição dos dados foi verificada utilizando o teste de normalidade de KolmogorovSmirnov. Para comparações entre grupos dos dados hemodinâmicos, empregou-se a ANOVA bifatorial, seguida da correção de Bonferroni, para determinar em quais momentos que a diferença foi significativa. Para comparações ao longo do tempo em cada grupo, os dados obtidos nos momentos 75, 90, 105 e 120 foram comparados entre si utilizando a ANOVA para amostras repetidas, seguida pelo teste de Tukey. Para comparações entre os grupos nas concentrações plasmáticas de propofol em cada momento, foi utilizado o teste de Mann Whitney. Os tempos de extubação, decúbito esternal e posição quadrupedal foram comparados por testes t não pareados. As diferenças foram consideradas significativas quando $\mathrm{P}<0,05$.

\section{RESULTADOS}

Quando comparada à anestesia com propofol, a anestesia com a associação propofollidocaína apresentou valores significativamente mais elevados de DC, IC, IS, PAS, PAM, PAD,
PVC, PAP, POAP e ETCO 2 (Tabela 1). Entretanto, diferenças entre momentos específicos foram detectadas somente para a variável POAP (aos 75 e 105 minutos tabela 1). Não houve diferenças significativas entre os grupos para FC, IRVS, IRVP, $f_{\mathrm{R}}$, temperatura e índice bispectral (Tabela 1). Os valores médios $( \pm \mathrm{DP})$ de $f_{\mathrm{R}}$ e temperatura observados em seis cães nos quatro momentos (75-120 minutos) foram $13 \pm 2 \mathrm{mpm}$ e $13 \pm 4 \mathrm{mpm}$ e $36,9 \pm 0,8^{\circ} \mathrm{C}$ e $37,0 \pm 0,9^{\circ} \mathrm{C}$, respectivamente, nos grupos $\mathrm{P}$ e PL. Houve redução significativa ao longo do tempo na PAP no grupo PL, sendo o valor aos 120 minutos menor que aos 75 minutos (tabela 1). Não houve diferenças ao longo do tempo nas demais variáveis em nenhum dos grupos. No grupo $\mathrm{P}$, ocorreu hipotensão (PAM $<60 \mathrm{mmHg}$ ) nos momentos 105 e 120 minutos em três cães e, em todos os momentos, em outro animal. No grupo PL, houve hipotensão em todos os momentos em um cão e apenas aos 120 minutos em outro animal.

As concentrações plasmáticas de propofol $\left(\mu \mathrm{g} \mathrm{mL}^{-1}\right)$ aos 75, 90, 105 e 120 minutos foram, respectivamente (medianas[intervalos interquartis]), de $5,8(2,5-6,9), 5,8(4,2-8,4), 5,7(4,5-7,8)$ e $6,1(5,1-$ $8,1)$ no grupo P e $3,3(2,6-4,0), 3,7(2,1-4,4), 3,4(2,6-$ $4,2)$ e $3,1(2,1-4,3)$ no grupo PL. Tais concentrações foram significativamente menores no grupo PL que as observados no grupo $\mathrm{P}$ aos $90(\mathrm{P}=0,026), 105$ $(\mathrm{P}=0,015)$ e $120(\mathrm{P}=0,015)$ minutos. As concentrações plasmáticas de lidocaína $\left(\mu \mathrm{g} \mathrm{mL}^{-1}\right)$ no grupo $\mathrm{PL}$ foram (medianas[intervalos interquartis]): 2,48(2,01-2,41), $2,41(2,12-2,45), 2,27(2,00-3,80)$ e $2,51(1,77-3,33)$, respectivamente, aos 75, 90, 105 e 120 minutos.

Os tempos decorridos em minutos até a extubação, decúbito esternal e posição quadrupedal foram (médias $\pm \mathrm{DP}$ ): $35 \pm 13$ e $44 \pm 27 ; 102 \pm 38$ e $128 \pm 74 ; 148 \pm 40$ e $157 \pm 76$, nos grupos P e PL, respectivamente, sem haver diferença significativa entre os grupos.

\section{DISCUSSÃO}

Em um estudo anterior, a administração da lidocaína nas mesmas doses empregadas aqui (bolus de $1,5 \mathrm{mg} \mathrm{kg}^{-1}$, infusão contínua de $0,25 \mathrm{mg}$ $\mathrm{kg}^{-1} \mathrm{~min}^{-1}$ ) reduziu a taxa de infusão mínima (TIM) do propofol em 18\% (TIM com propofol: $0,51 \mathrm{mg}$ $\mathrm{kg}^{-1} \mathrm{~min}^{-1}$; TIM com propofol-lidocaina: $0,42 \mathrm{mg} \mathrm{kg}^{-1}$ $\mathrm{min}^{-1}$ ). Entretanto, a redução na TIM do propofol pela lidocaína não foi estatisticamente significativa. Adicionalmente, não foram observados benefícios hemodinâmicos durante a anestesia com doses equivalentes a 1 TIM do propofol, associado à lidocaína, em comparação à anestesia com 1 TIM do 
Tabela 1 - Valores médios ( $\pm \mathrm{DP})$ da frequência cardíaca (FC), débito cardíaco (DC), índice cardíaco (IC), índice sistólico (IS), pressão arterial sistólica (PAS), média (PAM) e diastólica (PAD), pressão venosa central (PVC), índice de resistência vascular sistêmica (IRVS), pressão da artéria pulmonar (PAP), pressão de oclusão da artéria pulmonar (POAP), índice de resistência vascular pulmonar (IRVP), concentração expirada de dióxido de carbono ao final da expiração $\left(\mathrm{ETCO}_{2}\right)$ e índice bispectral (BIS) em 12 cães anestesiados com propofol (grupo $\mathrm{P}$ [n=6]: bolus de $6 \mathrm{mg} \mathrm{kg}^{-1}$ e infusão contínua de $1,25 \mathrm{mg} \mathrm{kg}^{-1} \mathrm{~min}^{-1}$ ) ou propofol associado à lidocaína (grupo PL [n=6]: bolus de $6 \mathrm{mg} \mathrm{kg}^{-1}$ de propofol e $1,5 \mathrm{mg} \mathrm{kg}^{-1}$ de lidocaína, e infusão de $1,0 \mathrm{mg} \mathrm{kg}^{-1} \mathrm{~min}^{-1} \mathrm{e}$ $0,25 \mathrm{mg} \mathrm{kg}^{-1} \mathrm{~min}^{-1}$, respectivamente).

\begin{tabular}{|c|c|c|c|c|c|c|}
\hline & Grupo & 75 & 90 & 105 & 120 & Valor de $\mathrm{P}^{\ddagger}$ \\
\hline \multirow{3}{*}{ FC (bat $\left.\min ^{-1}\right)$} & $P$ & $125 \pm 20$ & $117 \pm 16$ & $117 \pm 17$ & $115 \pm 14$ & \multirow{3}{*}{0,085} \\
\hline & & & & & & \\
\hline & PL & $110 \pm 17$ & $109 \pm 18$ & $111 \pm 16$ & $109 \pm 16$ & \\
\hline \multirow{2}{*}{$\mathrm{DC}\left(\mathrm{L} \mathrm{min}^{-1}\right)$} & $\mathrm{P}$ & $2,0 \pm 0,5$ & $2,0 \pm 0,6$ & $1,9 \pm 0,5$ & $1,6 \pm 0,6$ & \multirow{2}{*}{0,001} \\
\hline & PL & $2,4 \pm 0,6$ & $2,5 \pm 0,7$ & $2,5 \pm 0,6$ & $2,5 \pm 0,6$ & \\
\hline \multirow{2}{*}{$\mathrm{IC}\left(\mathrm{L} \mathrm{min}^{-1} \mathrm{~m}^{-2}\right)$} & $\mathrm{P}$ & $3,8 \pm 1,0$ & $3,8 \pm 1,1$ & $3,8 \pm 1,1$ & $3,2 \pm 1,2$ & \multirow{2}{*}{0,018} \\
\hline & PL & $4,4 \pm 1,1$ & $4,4 \pm 1,2$ & $4,5 \pm 1,1$ & $4,4 \pm 1,1$ & \\
\hline \multirow{2}{*}{ IS (mL bat $\left.{ }^{-1} \mathrm{~kg}^{-1}\right)$} & $\mathrm{P}$ & $1,37 \pm 0,36$ & $1,47 \pm 0,44$ & $1,47 \pm 0,44$ & $1,22 \pm 0,41$ & \multirow{2}{*}{0,006} \\
\hline & PL & $1,72 \pm 0,52$ & $1,78 \pm 0,50$ & $1,77 \pm 0,45$ & $1,77 \pm 0,43$ & \\
\hline \multirow{2}{*}{ PAS (mmHg) } & $\mathrm{P}$ & $98 \pm 23$ & $96 \pm 28$ & $85 \pm 33$ & $88 \pm 38$ & \multirow{2}{*}{0,011} \\
\hline & PL & $122 \pm 36$ & $122 \pm 37$ & $120 \pm 45$ & $117 \pm 47$ & \\
\hline \multirow{2}{*}{ PAM (mmHg) } & $\mathrm{P}$ & $73 \pm 21$ & $71 \pm 23$ & $62 \pm 26$ & $63 \pm 31$ & \multirow{2}{*}{0,008} \\
\hline & PL & $93 \pm 29$ & $92 \pm 29$ & $91 \pm 35$ & $87 \pm 36$ & \\
\hline \multirow{2}{*}{ PAD (mmHg) } & $\mathrm{P}$ & $60 \pm 20$ & $59 \pm 21$ & $50 \pm 24$ & $50 \pm 27$ & \multirow{2}{*}{0,009} \\
\hline & PL & $78 \pm 25$ & $77 \pm 25$ & $75 \pm 31$ & $71 \pm 31$ & \\
\hline \multirow{2}{*}{ PVC (mmHg) } & $\mathrm{P}$ & $3 \pm 3$ & $3 \pm 3$ & $3 \pm 3$ & $3 \pm 3$ & \multirow{2}{*}{0,004} \\
\hline & PL & $7 \pm 5$ & $7 \pm 5$ & $7 \pm 5$ & $7 \pm 5$ & \\
\hline \multirow{2}{*}{ IRVS (dinas seg ${ }^{-1} \mathrm{~cm}^{-5} \mathrm{~m}^{-2}$ ) } & $\mathrm{P}$ & $1515 \pm 339$ & $1505 \pm 482$ & $1318 \pm 485$ & $1522 \pm 490$ & \multirow{2}{*}{0,616} \\
\hline & PL & $1611 \pm 475$ & $1570 \pm 478$ & $1498 \pm 546$ & $1462 \pm 546$ & \\
\hline \multirow{2}{*}{ PAP (mmHg) } & $\mathrm{P}$ & $8 \pm 3$ & $8 \pm 3$ & $7 \pm 3$ & $8 \pm 2$ & \multirow{2}{*}{$<0,001$} \\
\hline & PL & $12 \pm 2 *$ & $11 \pm 2$ & $11 \pm 2$ & $10 \pm 2 *$ & \\
\hline \multirow{2}{*}{ POAP (mmHg) } & $\mathrm{P}$ & $1 \pm 1^{\dagger}$ & $1 \pm 1$ & $0 \pm 1^{\dagger}$ & $2 \pm 3$ & \multirow{2}{*}{$<0,001$} \\
\hline & PL & $4 \pm 4$ & $3 \pm 2$ & $4 \pm 2$ & $2 \pm 2$ & \\
\hline \multirow{2}{*}{ IRVP (dinas seg ${ }^{-1} \mathrm{~cm}^{-5} \mathrm{~m}^{-2}$ ) } & $\mathrm{P}$ & $189 \pm 123$ & $178 \pm 113$ & $173 \pm 110$ & $183 \pm 150$ & \multirow{2}{*}{0,168} \\
\hline & PL & $149 \pm 68$ & $141 \pm 38$ & $130 \pm 40$ & $149 \pm 36$ & \\
\hline \multirow{2}{*}{$\mathrm{ETCO}_{2}(\mathrm{mmHg})$} & $\mathrm{P}$ & $40 \pm 5$ & $38 \pm 5$ & $39 \pm 4$ & $38 \pm 2$ & \multirow{2}{*}{0,002} \\
\hline & PL & $44 \pm 5$ & $43 \pm 5$ & $43 \pm 5$ & $43 \pm 6$ & \\
\hline \multirow{2}{*}{ Índice Bispectral } & $\mathrm{P}$ & $46 \pm 23$ & $44 \pm 22$ & $43 \pm 22$ & $43 \pm 23$ & \multirow{2}{*}{0,551} \\
\hline & PL & $49 \pm 17$ & $49 \pm 17$ & $48 \pm 13$ & $45 \pm 12$ & \\
\hline
\end{tabular}

${ }^{\dagger}$ diferença significativa entre os grupos (Correção de Bonferroni); *diferença significativa entre os momentos 75 e 120 minutos para o grupo PL (teste de Tukey); ${ }^{\star}$ Valor de $P$ obtido pela ANOVA bifatorial para comparações entre grupos. Valores de $\mathrm{P}<0,05$ indicam diferença significativa entre os grupos, levando-se em consideração todos os momentos. Entretanto, na análise entre grupos, em momentos específicos (correção de Bonferroni), foram detectadas diferenças significativas somente para a variável POAP.

propofol isoladamente (MANNARINO et al., 2012). Durante a anestesia com anestésicos inalatórios halogenados, o emprego de dose equivalente à CAM, a qual corresponde à $\mathrm{DE}_{50}$ de um anestésico inalatório, resulta em anestesia em plano superficial. A anestesia em plano profundo é obtida com a utilização de concentrações anestésicas iguais ou acima de 2 CAM (STEFFEY \& MAMMA, 2007). Levando-se em consideração que a TIM representa a $\mathrm{DE}_{50}$ de um anestésico injetável, espera-se que a anestesia com dose igual ou acima de 2 TIM do propofol resulte em plano profundo. Baseado no estudo de MANNARINO 
et al. (2012), as taxas de infusão utilizadas no presente estudo foram de aproximadamente 2,4 vezes a TIM determinada com propofol isoladamente e propofol associado à lidocaína $\left(1,25 \mathrm{mg} \mathrm{kg} \mathrm{km}^{-1} \mathrm{~min}^{-1}\right.$ e $1,0 \mathrm{mg}$ $\mathrm{kg}^{-1} \mathrm{~min}^{-1}$, respectivamente). Essas doses foram escolhidas para comparar os efeitos hemodinâmicos durante a anestesia em plano profundo utilizando doses equipotentes de propofol.

As concentrações de lidocaína observadas no presente estudo ficaram abaixo dos valores que resultam em toxicidade em cães (valor máximo da mediana $=2,51 \mu \mathrm{g} \mathrm{mL}^{-1}$ aos 120 minutos) (LEMO et al., 2007) e aparentemente não resultaram em toxicidade cardiovascular, conforme demonstrado por valores mais elevados de IC, IS, PAS, PAM e PAD no grupo PL. Adicionalmente, as concentrações de lidocaína obtidas durante o regime de infusão empregado neste estudo (bolus de $1,5 \mathrm{mg} \mathrm{kg}^{-1}$ e infusão de $0,25 \mathrm{mg} \mathrm{kg}^{-1}$ $\mathrm{min}^{-1}$ ) podem ser consideradas eficazes, uma vez que concentrações de lidocaína a partir de $1,47 \mu \mathrm{g} \mathrm{mL}^{-1}$ causaram redução significativa na CAM do isoflurano em cães (VALVERDE et al., 2004).

Os valores de IC, IS, PAM, IRVS no grupo $\mathrm{P}$ foram menores e os valores de $\mathrm{FC}$ foram maiores do que os considerados normais para cães conscientes (HASKINS et al., 2005). Estudos anteriores realizados in vitro demonstraram que o propofol apresenta efeitos inotrópico e cronotrópico negativos em cães (NAGASHIMA et al., 1999, 2000). Em estudo realizado in vivo em cães, infusões de 0,25 a 2,0mg kg-1 $\mathrm{min}^{-1}$ causaram diminuições dose dependentes na pressão arterial e na contratilidade do miocárdio (PAGEL \& WARLTIER, 1993), incluindo redução no DC e na RVS durante as taxas de infusão maiores. Assim como no estudo de PAGEL \& WARLTIER (1993), no presente estudo, a redução da pressão arterial parece ter ocorrido pela diminuição no IC e na RVS. O fato do DC estar diminuído, apesar de valores de FC elevados, sugere que o propofol causou redução acentuada na contratilidade do miocárdio, a qual resultou em redução no IS.

Apesar dos valores de IC, IS, PAS, PAM e PAD não terem diferido significativamente entre os grupos P e PL nos momentos 75, 90, 105 e 120 (correção de Bonferroni), a análise de variância (ANOVA) revelou que os valores dessas variáveis, considerando todos os momentos, foram significativamente menores no grupo $\mathrm{P}$ do que no grupo PL. Um número maior de cães apresentou hipotensão no grupo $\mathrm{P}$ do que no grupo PL (4 versus 2 cães, respectivamente) e os valores médios de PAM no grupo $\mathrm{P}$ ficaram próximos ao limite considerado como hipotensão (PAM $<60 \mathrm{mmHg}$ ) aos
105 e 120 minutos. Relatou-se previamente que os efeitos cardiovasculares do propofol em cães são dose dependentes (PAGEL \& WARLTIER, 1993; NAGASHIMA et al., 1999, 2000). Os resultados deste estudo e dos estudos anteriores sugerem que a menor depressão cardiovascular observada no grupo PL ocorreu devido à menor taxa de infusão de propofol empregada neste grupo. Em um estudo recente, foram estudados os efeitos hemodinâmicos da ATIV com propofol $\left(0,51 \mathrm{mg} \mathrm{kg}^{-1} \mathrm{~min}^{-1}\right)$ e propofol associado à lidocaína $\left(0,41\right.$ e $0,25 \mathrm{mg} \mathrm{kg}^{-1} \mathrm{~min}^{-1}$, respectivamente). Apesar da redução de $18 \%$ do propofol nos cães que receberam propofol-lidocaína em relação ao grupo que recebeu propofol isoladamente, não se constataram benefícios hemodinâmicos da associação da lidocaína, já que houve redução na FC, IC e PAM nos dois grupos (MANNARINO et al., 2012). As doses empregadas no estudo anterior correspondem a 1 TIM, para o propofol isoladamente e associado à lidocaína. A manutenção da anestesia com 1 TIM de um anestésico injetável resulta em plano superficial de anestesia, enquanto que o emprego de doses iguais ou maiores do que duas vezes a $\mathrm{DE}_{50}$ (2 TIM) resultam em plano profundo de anestesia (SEAR \& PRYSROBERTS, 1979). Os resultados de MANNARINO et al., 2012 e do presente estudo sugerem que a redução na TIM do propofol pela lidocaína causa benefícios hemodinâmicos em anestesia profunda (2,4 TIM), mas não em anestesia superficial (1 TIM).

Em cães da raça Beagle, a administração de $0,4 \mathrm{mg} \mathrm{kg}^{-1} \mathrm{~min}^{-1}$ de propofol resultou em concentrações plasmáticas médias de aproximadamente 6,5 a $9,0 \mu \mathrm{g} \mathrm{mL}^{-1}$, decorridos 60 a 120 minutos de infusão (HALL et al., 1997), semelhantes às encontradas aqui (medianas de 5,8 a $6,1 \mu \mathrm{g} \mathrm{mL}^{-1}$ ) durante a infusão de propofol na dose de $1,25 \mathrm{mg} \mathrm{kg}^{-1} \mathrm{~min}^{-1}$. Tal discrepância entre os estudos pode ser explicada com base na grande variabilidade da farmacocinética do propofol em cães de diferentes raças e pesos (ZORAN et al., 1993; HALL et al., 1997).

$\mathrm{O}$ BIS tem sido utilizado como um indicador do grau de hipnose em cães (CAMPAGNOL et al., 2007; LOPES et al., 2008; MANNARINO et al., 2012). A administração do propofol sob a forma de infusão intravenosa contínua causou redução dose dependente no BIS em cães, mas não de forma linear (LOPES et al., 2008). Os valores de BIS aos 40 minutos de infusão foram significativamente menores durante a infusão na taxa de $0,8 \mathrm{mg} \mathrm{kg}^{-1} \mathrm{~min}^{-1}(72 \pm 10)$ do que durante infusões de 0,2 e $0,4 \mathrm{mg} \mathrm{kg}^{-1} \mathrm{~min}^{-1}$ ( $84 \pm 7$ e $83 \pm 9$, respectivamente). Em outro estudo realizado em cães, a infusão de propofol na dose de $0,5 \mathrm{mg} \mathrm{kg}^{-1} \mathrm{~min}^{-1}$ resultou em valores de BIS de 
68 a 75 (MANNARINO et al., 2012). No presente estudo, a infusão de propofol resultou em valores de BIS de 43 a 46 e 45 a 49 nos grupos P e PL, respectivamente. Esses valores são menores do que os observados durante a infusão de propofol nas doses de $0,5 \mathrm{mg} \mathrm{kg}^{-1} \mathrm{~min}^{-1}$ (MANNARINO et al., 2012) e $0,8 \mathrm{mg} \mathrm{kg}^{-1} \mathrm{~min}^{-1}$ (LOPES et al., 2008). Apesar das concentrações plasmáticas de propofol neste estudo terem sido menores do que as esperadas, os valores de BIS sugerem que a dose de propofol empregada foi suficiente para proporcionar plano profundo de anestesia nos grupos P e PL.

\section{CONCLUSÃO}

A administração de propofol na dose de $1,25 \mathrm{mg} \mathrm{kg}^{-1} \mathrm{~min}^{-1}$ isoladamente e $1,0 \mathrm{mg} \mathrm{kg}^{-1} \mathrm{~min}^{-1}$ em associação à lidocaína $\left(0,25 \mathrm{mg} \mathrm{kg}^{-1} \mathrm{~min}^{-1}\right)$ causa plano profundo de anestesia em cães. A manutenção da anestesia em plano profundo com lidocaínapropofol causa menor depressão cardiovascular do que a anestesia com dose equipotente de propofol isoladamente, conforme demonstrado por valores mais elevados de IC, IS e PAM, o que sugere que a lidocaína deve ser associada ao propofol durante a anestesia em plano profundo, para minimizar a depressão cardiovascular.

\section{AGRADECIMENTOS}

À Fundação de Amparo à Pesquisa do Estado de São Paulo (FAPESP) pela bolsa concedida.

\section{FONTES DE AQUISIÇÃO}

a - Diprivan, AstraZeneca do Brasil Ltda.

b - Xylocaína 1\% sem vasoconstritor, Astra Química e Farmacêutica Ltda.

c - Bomba de Infusão de Seringa Digipump SR 2000, Digicare.

d - Samurai III, Takaoka.

e - AS/3 Anaesthesia Monitor, DatexËngstrom

f - Transdutor de Pressão TruWave, Baxter Healthcare Corporation.

g - Intro-Flex, Kit Introdutor Percutâneo, Baxter Healthcare Corporation.

h - Criticath Thermodilution Catheter, Ohmeda.

i - A-2000 Standard System; Aspect Medical Systems.

\section{COMITÊ DE ÉTICA}

Aprovado pela Comissão de Ética no Uso de Animais (CEUA) da Instituição (protocolo 334).

\section{REFERÊNCIAS}

CAMPAGNOL, D. et al. Use of bispectral index to monitor depth of anesthesia in isoflurane-anesthetized dogs. American Journal of Veterinary Research, v.68, n.12, p.1300-1307, 2007.
DOHERTY, T.J.; FRAZIER, D.L. Effect of intravenous lidocaine on halothane minimum alveolar concentration in ponies. Equine Veterinary Journal, v.30, p.300-303, 1998.

FRÖLICH, M.A. et al. The effect of propofol on thermal pain perception. Anesthesia and Analgesia, v.100, p.481-496, 2005.

HALL, L. et al. Disposition of propofol after medetomidine premedication in beagle dogs. Veterinary Anaesthesia and Analgesia, v.24, n.1, p.23-29, 1997.

HASKINS, S. et al. Reference cardiopulmonary values in normal dogs. Comparative Medicine, v.55, n.2, p.156-161, 2005.

KEEGAN, R.D.; GREENE, S.A. Cardiovascular effects of a continuous two-hour propofol infusion in dogs. Comparison with isoflurane anesthesia. Veterinary Surgery, v.22, n.6, p.537-543, 1993.

LEMO, N. et al. Determination of the toxic dose of lidocaine in dogs and its corresponding serum concentration. Veterinary Record, v.160, p.374-375, 2007.

LOPES, P.C.F. et al. Bispectral index in dogs at three intravenous infusion rates of propofol. Veterinary Anaesthesia and Analgesia, v.35, n.3, p.228-231, 2008.

MANNARINO, R. et al. Minimum infusion rate and hemodynamic effects of propofol, propofol-lidocaine and propofol-lidocaineketamine in dogs. Veterinary Anaesthesia and Analgesia, v.39, n.2, p.160-173, 2012.

NAGASHIMA, Y. et al. Cardiac effects of propofol and its interaction with autonomic nervous system in isolated, cross-circulated canine atria. Journal of Anesthesia, v.13, n.1, p.34-39, 1999.

NAGASHIMA, Y. et al. Propofol decreases contractility of isolated blood-perfused left ventricular muscle in the dog. Journal of Anesthesia, v.14, n.1, p.45-47, 2000.

PAGEL, P.S.; WARLTIER, D.C. Negative inotropic effects of propofol as evaluated by the regional preload recruitable stroke work relationship in chronically instrumented dogs. Anesthesiology, v.78, p.100-108, 1993.

SEAR, J.W.; PRYS-ROBERTS, C. Dose-related haemodynamic effects of continuous infusions of Althesin in man. British Journal of Anaesthesia, v.51, p.867-893, 1979.

STEFFEY, E.P.; MAMA, K.R. Inhalation anesthetics. In: TRANQUILLI, W.J. et al. Lumb \& Jones' veterinary anesthesia and analgesia. Ames: Blackwell Publishing, 2007. Cap.13, p.355-393.

TAKECHI, K. et al. The antinociceptive and antihyperalgesic effects of topical propofol on dorsal horn neurons in the rat. Anesthesia and Analgesia, v.116, p.932-938, 2013.

VALVERDE, A. et al. Effect of lidocaine on the minimum alveolar concentration of isoflurane in dogs. Veterinary Anaesthesia and Analgesia, v.31, n.4, p.264-271, 2004.

VIEIRA, F.A.F. Comparação entre propofol e propofol/ quetamina na indução e manutenção anestésica por infusão contínua intravenosa após medicação pré-anestésica com levomepromazina em cães. 1999. 123f. Dissertação (Mestrado em Medicina Veterinária) - Faculdade de Medicina Veterinária e Zootecnia, Universidade Estadual Paulista, SP.

ZORAN, D.L. et al. Pharmacokinetics of propofol in mixedbreed dogs and greyhounds. American Journal of Veterinary Research, v.54, n.5, p.755-760, 1993.

Ciência Rural, v.44, n.2, fev, 2014. 